\title{
The Exact Solitary Wave Solutions in Continuity Equation of the One-Dimensional Granular Crystals of Elastic Spheres
}

\author{
Zhiguo Liu' ${ }^{1}$ Jinliang Zhang² \\ ${ }^{1}$ Institute of Engineering Mechanics, Beijing Jiaotong University, Beijing, China \\ ${ }^{2}$ School of Mathematics and Statistics, Henan University of Science and Technology, Luoyang, China \\ Email: 15115257@bjtu.edu.cn
}

How to cite this paper: Liu, Z.G. and Zhang, J.L. (2019) The Exact Solitary Wave Solutions in Continuity Equation of the One-Dimensional Granular Crystals of Elastic Spheres. Journal of Applied Mathematics and Physics, 7, 2760-2766.

https://doi.org/10.4236/jamp.2019.711189

Received: October 9, 2019

Accepted: November 10, 2019

Published: November 13, 2019

\begin{abstract}
In this paper, we reduced the governing equation describing the one-dimensional granular crystals of elastic spheres to a continuous equation by small deformation and long wave approximation. Then, the G'/G-expansion method is applied to this continuous equation, and the exact solitary wave solutions with arbitrary parameters are obtained. Compared with other papers, the solutions obtained in this paper are more extensive and contains more parameters. The simultaneous existence of exact solitary wave solutions can help us study the propagation of shock waves in one-dimensional granular crystals of elastic spheres. At the same time, it has important theoretical significance in nondestructive testing with non-linear wave.
\end{abstract}

\section{Keywords}

Granular Crystals of Elastic Spheres, G'/G-Expansion Method, Solitary Wave, Shock Waves

\section{Introduction}

In recent years, the study of the propagation of highly nonlinear solitary waves in granular materials has drawn considerable attention from the scientific community [1] [2] [3] [4].

A solitary wave was shown to be an ideal method for transferring vibrational excitations [5]. Elastic spherical chain is an ideal experimental device for studying nonlinear science. Because the spherical chain is in a strong nonlinear state under a small precompression, the spherical chain is in a weak nonlinear state under a strong precompression. Such tunability is valuable not only for studies 
of the basic physics of granular lattices but also in potential engineering applications, such as energy trapping [6], energy harvesting [7], nonlinear waves sensor technology [8], acoustic lenses [9], acoustic diodes [10] and switches [11], and sound scramblers [12], and more.

The dynamic properties of one-dimensional granular crystals have been extensively studied, using analytical, numerical, and experimental methods. In Reference [1] [13], the numerical solitary wave solutions were obtained in a chain of granular spheres. In Reference [2], the approximate analytic dark solitary wave solutions were obtained in a chain of uncompressed elastic beads. Moreover, In Reference [14] the approximate bright and dark solitary wave solutions were obtained in the chain of elastic spheres.

In the present work, we use G'/G-expansion method [15] [16] [17] [18] [19] to investigate the eigensolutions of elastic spherical chains.

\section{The Continuous Equation of One-Dimensional Granular Crystals of Elastic Spheres}

A granular crystal of elastic spheres compressed by a static force $F_{0}$ is considered, as shown in Figure 1. For this elastic sphere, $m=4 / 3 \pi R^{3} \rho_{0}$, where $m$ is the mass of the elastic spheres, Moreover, it is assumed that the one-dimensional granular crystals is subjected to a static constant force $F_{0}$, resulting in an initial displacement $\delta_{0}$ between neighboring particle centers.

Using the dynamic equilibrium condition, the equation describing the motion of the one-dimensional granular crystals of elastic spheres can be derived as:

$$
\ddot{u}_{i}=A\left(\delta_{0}-u_{i}+u_{i-1}\right)^{\frac{3}{2}}-A\left(\delta_{0}-u_{i+1}+u_{i}\right)^{\frac{3}{2}},
$$

where $A=\frac{E \sqrt{2 R}}{3\left(1-v^{2}\right)}$ is the Hertzian constant determined by material properties of the beads and the radius of the contact curvature, $E$ is the Young's modulus, $\rho_{0}$ is the density of the sphere material, $R$ and $v$ are the sphere radius and Poisson's ratio.

If the force between the elastic spheres is a small nonlinear force and the static compression at the initial time is greater than the interparticle compression, we have

$$
\frac{\left|u_{i-1}-u_{i}\right|}{\delta_{0}} \ll 1
$$

Then from Equation (1), we have (of Equation (2.2) in [1])

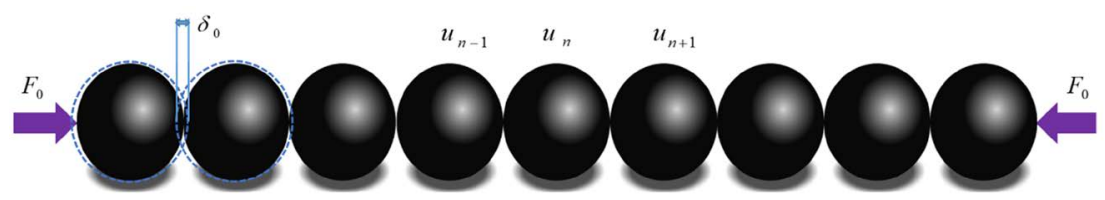

Figure 1. One-dimensional granular crystals of elastic spheres compressed by a static force $F_{0}$. 


$$
\ddot{u}_{i}=\frac{3}{2} A \delta_{0}^{\frac{1}{2}}\left(u_{i+1}-2 u_{i}+u_{i-1}\right)+\frac{3}{8} A \delta_{0}^{-\frac{1}{2}}\left(u_{i+1}-2 u_{i}+u_{i-1}\right)\left(u_{i-1}-u_{i+1}\right)
$$

In the long-wave approximation, Equation (3) can be written as the continuation form:

$$
u_{t t}-c_{0}^{2} u_{x x}=-\varepsilon u_{x} u_{x x}+2 c_{0} \gamma u_{x x x x}-\frac{6 \varepsilon \gamma}{10 c_{0}} u_{x x x x x}-\frac{36 \varepsilon \gamma}{c_{0}} u_{x x} u_{x x x}-\frac{18 \varepsilon \gamma}{c_{0}} u_{x} u_{x x x x}
$$

where $c_{0}^{2}=A \delta_{0}^{1 / 2} 6 R^{2}, \gamma=c_{0} R^{2} / 6, \varepsilon=c_{0}^{2} R / \delta_{0}$.

Ignoring the infinitely small quantities of the fifth order, we obtain

$$
u_{t t}-c_{0}^{2} u_{x x}-2 c_{0} \gamma u_{x x x x}+\varepsilon u_{x} u_{x x}=0
$$

Next, we use the G'/G-expansion method to solve Equation (5).

\section{The Exact Solutions to Equation (5)}

Firstly, the traveling wave transformation is performed

$$
u(\mathrm{x}, \mathrm{t})=u(\xi), \xi=k \mathrm{x}-\omega \mathrm{t}+\xi_{0}
$$

where $k$ and $\omega$ are undetermined constants and $\xi_{0}$ is a constant.

When Equation (6) is brought into Equation (5), the following ordinary differential equations are obtained.

$$
\left(\omega^{2}-c_{0}^{2} k^{2}\right) u_{\xi}-2 c_{0} \gamma k^{4} u_{\xi \xi \xi \xi}+\varepsilon k^{3} u_{\xi} u_{\xi \xi}
$$

By integrating Equation (7) once and taking the integral constant as zero, we can get the result

$$
\left(\omega^{2}-c_{0}^{2} k^{2}\right) u-2 c_{0} \gamma k^{4} u_{\xi \xi \xi}+\frac{\varepsilon k^{3}}{2}\left(u_{\xi}\right)^{2}=0
$$

Assuming that the solution of Equation (8) is

$$
u(\xi)=\sum_{i=0}^{m}\left\{a_{i}\left(\frac{G^{\prime}}{G}\right)^{i}+b_{i} \sqrt{\sigma\left[\frac{1+\left(\frac{G^{\prime}}{G}\right)^{2 i}}{\mu}\right]}\right\}
$$

where $m, a_{i}, b_{i}$ are constant to be determined, $\sigma= \pm 1, a_{i} b_{i}=0, a_{i}^{2}+b_{i}^{2} \neq 0$, $G=G(\xi)$ satisfies the following second order linear ordinary differential equations

$$
G^{\prime \prime}(\xi)+\mu G(\xi)=0
$$

By solving Equation (10), we can get

$$
\frac{G^{\prime}\left(\xi_{n}\right)}{G\left(\xi_{n}\right)}=\left\{\begin{array}{l}
\sqrt{-\mu}\left(\frac{C_{1} \sinh \left(\sqrt{-\mu} \xi_{n}\right)+C_{2} \cosh \left(\sqrt{-\mu} \xi_{n}\right)}{C_{2} \sinh \left(\sqrt{-\mu} \xi_{n}\right)+C_{1} \cosh \left(\sqrt{-\mu} \xi_{n}\right)}\right), \mu<0 \\
\sqrt{\mu}\left(\frac{-C_{1} \sin \left(\sqrt{\mu} \xi_{n}\right)+C_{2} \cos \left(\sqrt{\mu} \xi_{n}\right)}{C_{2} \sin \left(\sqrt{\mu} \xi_{n}\right)+C_{1} \cos \left(\sqrt{\mu} \xi_{n}\right)}\right), \mu>0 \\
\frac{C_{1}}{C_{1} \xi_{n}+C_{2}}, \mu=0
\end{array}\right.
$$


where $C_{1}, C_{2}$ are constants.

From Equation (10), it can be obtained

$$
\begin{gathered}
u_{\xi}=-m a_{m}\left(\frac{G^{\prime}}{G}\right)^{m+1}+\cdots \\
\left(u_{\xi}\right)^{2}=m^{2} a_{m}^{2}\left(\frac{G^{\prime}}{G}\right)^{2 m+2}+\cdots \\
u_{\xi \xi \xi}=-m(m+1)(m+2) a_{m}\left(\frac{G^{\prime}}{G}\right)^{m+3}+\cdots
\end{gathered}
$$

Substituting Equation (12) and Equation (14) into Equation (8) and applying the principle of homogeneous balance yield

$$
2 m+2=m+3
$$

It can be obtained from Equation (15) that $m=1$, so Equation (9) can be written as

$$
u(\xi)=a_{0}+a_{1}\left(\frac{G^{\prime}}{G}\right)+b_{1} \sqrt{\sigma\left[\frac{1+\left(\frac{G^{\prime}}{G}\right)^{2}}{\mu}\right]}
$$

Substitute Equation (16) into Equation (8), merging the same power terms of $\left(G^{\prime} / G\right)$ and making the coefficients of these same power terms zero, the following equations can be obtained

$$
\begin{gathered}
-a_{1} \mu\left(\omega^{2}-c_{0}^{2} k^{2}\right)+4 a_{1} \mu^{2} c_{0} \gamma k^{4}+\frac{1}{2} \varepsilon k^{3} a_{1}^{2} \mu^{2}=0 \\
-a_{1}\left(\omega^{2}-c_{0}^{2} k^{2}\right)+16 a_{1} \mu c_{0} \gamma k^{4}+\varepsilon k^{3} a_{1}^{2} \mu=0 \\
12 a_{1} c_{0} \gamma k^{4}+\frac{1}{2} \varepsilon k^{3} a_{1}^{2}=0
\end{gathered}
$$

or

$$
\begin{aligned}
& 8 c_{0} \gamma k^{4}\left(\omega^{2}-c_{0}^{2} k^{2}-2 c_{0} \gamma k^{4}\right)=0 \\
& \left(\omega^{2}-c_{0}^{2} k^{2}-2 c_{0} \gamma k^{4}\right)^{2}=-\frac{b_{1}^{2} \varepsilon^{2} k^{6}}{12}
\end{aligned}
$$

Solving algebraic Equation (17)-(21), we can get

$$
a_{1}=-24 \frac{c_{0} \gamma k}{\varepsilon}, \omega^{2}=c_{0}^{2} k^{2}+8 \mu c_{0} \gamma k^{4}, b_{1}=0
$$

when $\mu<0$, the following hyperbolic function solutions can be obtained

$$
u(\xi)=a_{0}+24 \frac{c_{0} \gamma k \mu}{\varepsilon}\left(\frac{C_{1} \sinh (-\mu \xi)+C_{2} \cosh (-\mu \xi)}{C_{1} \cosh (-\mu \xi)+C_{2} \sinh (-\mu \xi)}\right)
$$

When $C_{1}, C_{2}$ take specific constants, Equation (23) can degenerate into solitary wave solution. For example, when $C_{1} \neq 0, C_{2}=0$, Equation (23) degenerates to: 


$$
u(\xi)=a_{0}+E \tanh (-\mu \xi)
$$

where $E=24 \frac{c_{0} \gamma k \mu}{\varepsilon}, \quad \xi=k \mathrm{x}-\omega \mathrm{t}+\xi_{0}, \quad \xi_{0} \quad$ is an arbitrary constant, $\omega^{2}=c_{0}^{2} k^{2}+8 \mu c_{0} \gamma k^{4}, C_{1}, C_{2}$ are constants.

The displacement profiles of the of the exact single solitary wave solution Equation (24), are shown in Figure 2. It is shown that the displacement of the exact single solitary waves is dark solitons.

\section{Discussion and Conclusions}

In this paper, the continuous equation of one-dimensional granular crystals of elastic spheres is derived; and the G'/G-expansion method is applied to this continuous equation, the hyperbolic function solitary wave solutions, trigonometric function periodic wave solutions and rational wave solutions with arbitrary parameters are obtained. Solitary wave solution Equation (24) is a special form of hyperbolic function solution Equation (23), and the form of the solitary wave solutions obtained in [2] [14] are the same, but the solutions in this paper are more extensive and contains more parameters. From the results of this paper, we can see that there are exact solitary wave solutions in one-dimensional granular crystals of elastic spheres. Furthermore, the existence of solitary wave solutions has important theoretical significance for us to study the propagation of shock waves in one-dimensional granular crystals of elastic spheres. At the same time, it has important theoretical significance in nondestructive testing with non-linear wave. These studies will be published in our follow-up research.

\section{Acknowledgements}

This work is supported by the National Natural Science Foundation of China (Grant no.51675161). The authors express their sincere thanks to the referee for valuable suggestions.

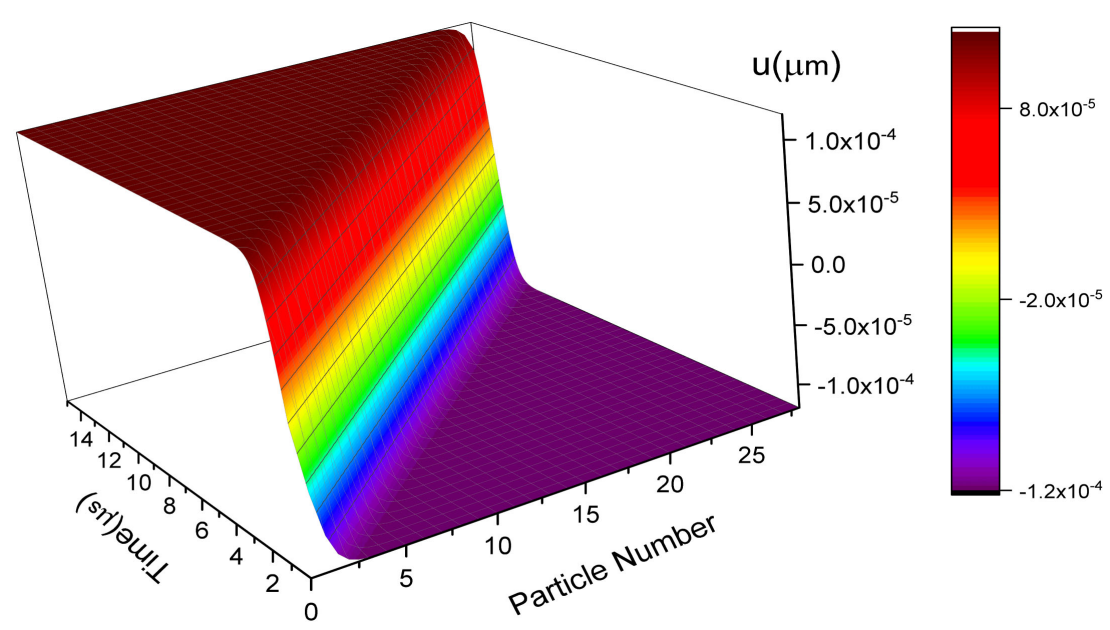

Figure 2. Evolution of the exact single solitary wave solution Equation (24) in the space-time domain with $a_{0}=0, E=-1.2 \times 10^{-4}(\mu S), C_{1}=1, C_{2}=2, k_{1}=1\left(m^{-1}\right)$. 


\section{Conflicts of Interest}

The authors declare no conflicts of interest regarding the publication of this paper.

\section{References}

[1] Nesterenko, V.F. (1983) Propagation of Nonlinear Compression Pulses in Granular Media. Journal of Applied Mechanics and Technical Physics, 24, 733-743. https://doi.org/10.1007/BF00905892

[2] Sen, S., Hong, J., Bang, J., et al. (2008) Solitary Waves in the Granular Chain. Physics Reports, 462, 21-66. https://doi.org/10.1016/j.physrep.2007.10.007

[3] Sen, S., Manciu, M., Sinkovits, R.S., et al. (2001) Nonlinear Acoustics in Granular Assemblies. Granular Matter, 3, 33-39. https://doi.org/10.1007/s100350000067

[4] Nesterenko, V. (2013) Dynamics of Heterogeneous Materials. Springer Science \& Business Media.

[5] Lupichev, L.N., Savin, A.V. and Kadantsev, V.N. (2015) Synergetics of Molecular Systems. Springer. https://doi.org/10.1007/978-3-319-08195-3

[6] Daraio, C., Nesterenko, V.F., Herbold, E.B., et al. (2006) Energy Trapping and Shock Disintegration in a Composite Granular Medium. Physical Review Letters, 96, Article ID: 58002. https://doi.org/10.1103/PhysRevLett.96.058002

[7] Doney, R. and Sen, S. (2006) Decorated, Tapered, and Highly Nonlinear Granular Chain. Physical Review Letters, 97, Article ID: 155502. https://doi.org/10.1103/PhysRevLett.97.155502

[8] Khatri, D., Daraio, C. and Rizzo, P. (2008) Highly Nonlinear Waves' Sensor Technology for Highway Infrastructures. International Society for Optics and Photonics. https://doi.org/10.1117/12.775848

[9] Spadoni, A. and Daraio, C. (2010) Generation and Control of Sound Bullets with a Nonlinear Acoustic Lens. Proceedings of the National Academy of Sciences, 107, 7230-7234. https://doi.org/10.1073/pnas.1001514107

[10] Liang, B., Yuan, B. and Cheng, J. (2009) Acoustic Diode: Rectification of Acoustic Energy Flux in One-Dimensional Systems. Physical Review Letters, 103, Article ID: 104301. https://doi.org/10.1103/PhysRevLett.103.104301

[11] Boechler, N., Theocharis, G. and Daraio, C. (2011) Bifurcation-Based Acoustic Switching and Rectification. Nature Materials, 10, 665.

https://doi.org/10.1038/nmat3072

[12] Nesterenko, V.F., Daraio, C., Herbold, E.B., et al. (2005) Anomalous Wave Reflection at the Interface of Two Strongly Nonlinear Granular Media. Physical Review Letters, 95, Article ID: 158702. https://doi.org/10.1103/PhysRevLett.95.158702

[13] Lazaridi, A.N. and Nesterenko, V.F. (1985) Observation of a New Type of Solitary Waves in a One-Dimensional Granular Medium. Journal of Applied Mechanics and Technical Physics, 26, 405-408. https://doi.org/10.1007/BF00910379

[14] Liu, Z., Wang, Y. and Huang, G. (2019) Solitary Waves in a Granular Chain of Elastic Spheres: Multiple Solitary Solutions and Their Stabilities. Physical Review E, 99, Article ID: 62904. https://doi.org/10.1103/PhysRevE.99.062904

[15] Wang, M. (1995) Solitary Wave Solutions for Variant Boussinesq Equations. Physics Letters A, 199, 169-172. https://doi.org/10.1016/0375-9601(95)00092-H

[16] Wang, M. (1996) Exact Solutions for a Compound KdV-Burgers Equation. Physics Letters A, 213, 279-287. https://doi.org/10.1016/0375-9601(96)00103-X 
[17] Wang, M., Zhou, Y. and Li, Z. (1996) Application of a Homogeneous Balance Method to Exact Solutions of Nonlinear Equations in Mathematical Physics. Physics Letters A, 216, 67-75. https://doi.org/10.1016/0375-9601(96)00283-6

[18] Zhang, J.-L. and Liu, Z.-G. (2011) Exact Solutions of Discrete Complex Cubic Ginzburg-Landau Equation and Their Linear Stability. Communications in Theoretical Physics, 56, Article ID: 1111. https://doi.org/10.1088/0253-6102/56/6/24

[19] Zhang, J., Liu, Z., Li, S., et al. (2012) Solitary Waves and Stable Analysis for the Quintic Discrete Nonlinear Schrödinger Equation. Physica Scripta, 86, Article ID: 15401. https://doi.org/10.1088/0031-8949/86/01/015401 